VIII ${ }^{\text {èmes }}$ Journées Nationales Génie Civil - Génie Côtier, Compiègne, 7-9 septembre 2004

\title{
Simulations numériques et expérimentales d'un soliton se propageant sur une plage inclinée.
}

\author{
Pierre Lubin ${ }^{(\mathrm{a})}$, Stéphane Vincent ${ }^{(\mathrm{a})}$, Stéphan T. Grilli ${ }^{(\mathrm{b})}$, Richard W. Gilbert ${ }^{(\mathrm{b})}$, \\ Déborah Drevard ${ }^{(\mathrm{c})}$, Vincent Rey ${ }^{(\mathrm{c})}$, Philippe Fraunié ${ }^{(\mathrm{c})}$, Richard Marcer ${ }^{(\mathrm{d})}$, \\ Dominique Astruc ${ }^{(\mathrm{e})}$, Dominique Legendre ${ }^{(\mathrm{e})}$, Marie Duval $^{(\mathrm{e})}$, Olivier \\ Kimmoun $^{(\mathrm{f})}$, Hubert Branger ${ }^{(\mathrm{g})}$, Stéphan Guignard ${ }^{(\mathrm{g})}$, Stéphane Abadie ${ }^{(\mathrm{h})}$.

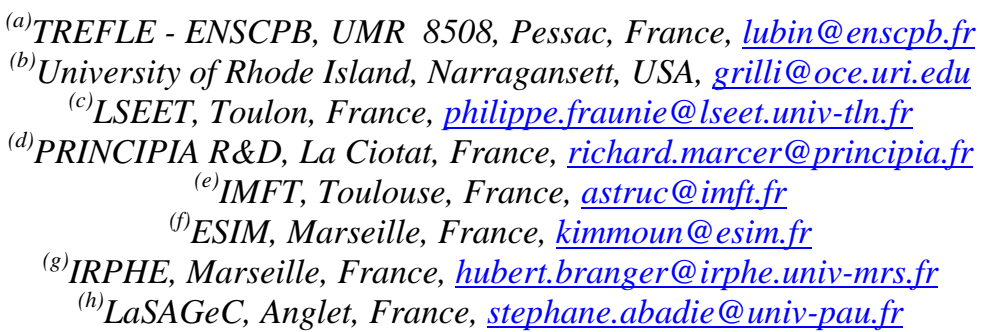

\section{Résumé}

Des simulations expérimentales de solitons se propageant et déferlant sur une plage, réalisées dans le canal à houle de l'ESIM à Marseille, sont présentées. Ces expériences de laboratoire feront l'objet d'un futur exercice de comparaisons pour des codes numériques, basés sur la résolution des équations de Navier-Stokes en formulation diphasique. Les laboratoires composant le groupe de travail, les différentes méthodes numériques, ainsi que les codes utilisés, sont exposés. L'objectif est d'aboutir à une description plus fine du phénomène du déferlement et à une meilleure compréhension des processus mis en jeu dans la zone d'impact. Ce travail est soutenu financièrement par le programme CNRS - PATOM.

\section{Abstract}

Experiments simulating shoaling and breaking solitary waves on slopes, performed at the ESIM's laboratory, are presented. The results obtained from these experiments will be used for validating and comparing fluid dynamic models, based on the Navier-Stokes equations adapted to two-phase flows. The laboratories which participate and the numerical methods are detailed. The aim of this work is to be able to give an accurate description of the breaking phenomenon and to reach a better understanding of the processes acting in the impact area. This work is financially supported by the CNRS - PATOM program.

Mots-clés: déferlement, simulations expérimentales, simulations numériques, méthodes VOF, méthodes BEM, Navier - Stokes.

\section{Introduction}

Dans le cadre du thème «Hydrodynamique des vagues en zone de déferlement» du Programme National sur l'Atmosphère et l'Océan Multi-échelles (PATOM), géré par l'Institut National des Sciences de l'Univers (INSU), un exercice de validations et de comparaisons de codes numériques a réuni plusieurs laboratoires. Des expériences de déferlement d'ondes solitaires en canal à houle ont été menées en laboratoire à l'Ecole Supérieure d'Ingénieurs de Marseille (ESIM). 
Ces expériences offrent ainsi des données de validation et de comparaison pour les codes Navier-Stokes, en terme de profils de surface libre et de champs de vitesse. Les codes numériques développés par l'Institut de Mécanique des Fluides (IMFT), l'Institut de Recherche sur les Phénomènes Hors Equilibre (IRPHE), le laboratoire Transferts, Ecoulements, Fluides, Energétique (TREFLE) et le Laboratoire de Sondages Electromagnétiques de l'Environnement Terrestre (LSEET) sont utilisés pour reproduire le déferlement des ondes solitaires. Les modèles de type Navier-Stokes sont à même de décrire la turbulence induite par le déferlement et à modéliser l'écoulement après impact du jet, les données expérimentales servant de référence pour les comparaisons. Des difficultés liées aux types d'ondes générées expérimentalement sont apparues à l'initialisation des simulations. En effet, il a été mis en évidence que les contraintes physiques du batteur (vitesse, accélération et décélération) n'ont pas permis la génération de solitons parfaits de type Tanaka (1986), ni d'ondes correspondant aux différentes théories analytiques disponibles $\left(1^{\text {er }}\right.$ ou $3^{\text {ème }}$ ordre d'approximation). Les codes Navier-Stokes ne pouvaient donc pas être initialisés à partir de solutions simples. La génération du soliton, ainsi que la propagation de la vague jusqu'au retournement de celle-ci, ont alors été simulées par le «canal à houle numérique » de l'Université de Rhodes Island, basé sur un code de type Méthode aux Eléments Frontières (BEM), afin de fournir les conditions initiales de champs de vitesses et de surface libre aux codes Navier-Stokes.

La première partie décrit les expériences réalisées dans le laboratoire de l'ESIM et le dispositif associé. Des images de déferlement de différentes ondes sont présentées. La seconde partie introduit les différentes méthodes numériques utilisées durant cet exercice de comparaison. Les différents laboratoires présentent leurs codes, résument leurs particularités et illustrent leurs potentialités. En conclusion, les travaux futurs sont introduits, le but de cet article étant de présenter les différents acteurs participant à l'exercice de comparaison, ainsi que les modalités de l'exercice de comparaison. Les futures simulations seront réalisées à partir du couplage entre les champs de données issus du code BEM et les codes NavierStokes.

\section{$\underline{\text { 2.Présentation des simulations expérimentales }}$}

Les expériences ont été conduites dans le canal à houle de l'ESIM à Marseille dans le but de mesurer à partir de différentes techniques les caractéristiques cinématiques et dynamiques de solitons se propageant et déferlant sur une plage. Une coupe schématique du canal et de l'appareillage de mesure est présentée sur la figure 1. Le canal a une longueur de $16,8 \mathrm{~m}$ et une largeur de $0,65 \mathrm{~m}$. Les parois sont en verre. La plage est constituée d'une plaque en PVC de $9 \mathrm{~m}$ de long et de pente $1: 15$, supportée tous les $50 \mathrm{~cm}$ par des étais rigides. A plus grande profondeur, une plaque en PVC de 1,44 $\mathrm{m}$ de long et de pente $1: 6$ est accolée à la plage principale. Une description plus détaillée de la plage avec les différentes cotes se trouve dans Grilli et al. (2004).

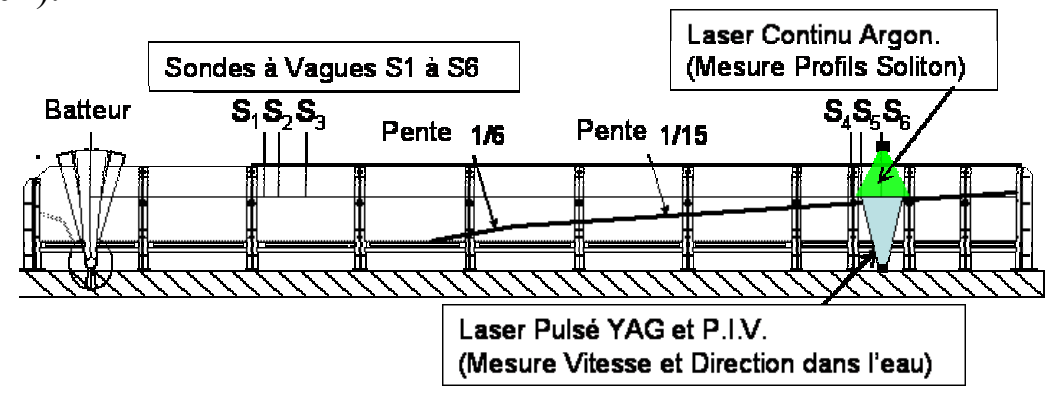

Figure 1 : configuration expérimentale dans le canal de l'ESIM. 
Les solitons ont été générés en envoyant au batteur volet pivotant autour d'un axe, un signal analogique correspondant à un seul déplacement rapide du volet de l'arrière vers l'avant. Le batteur est déplacé tout d'abord très lentement vers l'arrière, puis après une pause, il se déplace violemment vers l'avant, et est ensuite freiné. Les contraintes physiques du batteur (vitesse, accélération et décélération) ne permettent pas la génération d'un soliton parfait de type Tanaka (1986) (voir Grilli et al. (2004)). Nous avons généré différents solitons, de différentes hauteurs pour différentes profondeurs d'eau. Six sondes à vagues résistives permettent d'avoir les évolutions temporelles des hauteurs d'eau au passage du soliton, avant la plage, et sur la plage pendant la phase de déferlement. Les profils spatiaux de l'interface ont été mesurés à l'aide d'une caméra numérique SONY placée sur le côté et visant horizontalement la surface de l'eau à une cadence de 50 images par seconde. L'interface est détectée automatiquement, grâce au fort contraste de couleur engendré par la nappe de lumière du laser argon placé au dessus du canal et éclairant l'eau tintée de fluorescéine.

Un système complet de mesure de champs de vitesse par imagerie de particules (P.I.V) (Adrian, 1991, pour une revue) a été utilisé. Un laser double cavité pulsé Nd-YAg, muni d'un bras articulé placé au dessous du canal, envoie des séries de couples d'impulsions de lumière laser de forte intensité. L'eau est ensemencée de particules de verre recouvertes d'argent, de 8 $\mu \mathrm{m}$ de diamètre, de flottabilité neutre. Une caméra Kodak Megaplus, placée sur le côté, à l'altitude de l'eau au repos, et visant horizontalement la paroi latérale du canal, prend des couples d'images de 1008x1018 pixels, de façon synchronisée avec les impulsions lasers. La durée entre deux impulsions d'un même couple est de $5 \mathrm{~ms}$. La durée entre deux couples successifs est de $100 \mathrm{~ms}$. Le mouvement des particules éclairées est analysé ensuite par intercorrélation entre différentes parties d'images. Les particularités du logiciel utilisé, adapté en particulier aux mouvements fluides avec des gradients importants, sont exposées dans Meunier et Leweke (2003). Les principales difficultés ont été le masquage automatique de l'interface et des parties situées dans l'air. Le masquage est nécessaire pour ne pas induire des erreurs sur le calcul des vitesses de particules. L'erreur relative en vitesse est de l'ordre de $17 \%$, et l'erreur absolue en direction est de l'ordre de 7 degrés (Kimmoun et al., 2004). Il s'agit de valeurs moyennes. Localement, si les conditions d'éclairage sont mauvaises, ou si la présence de bulles induit des saturations d'intensité, les erreurs peuvent être plus importantes.

Des résultats de champ de vitesse PIV sont montrés sur la figure 2. Un champ de vitesse PIV étant issu d'un traitement de deux images prises à $100 \mathrm{~ms}$ d'intervalle, deux profils sont visibles sur la figure 2. Pour des raisons de clarté, un vecteur sur quatre est représenté. Certaines vitesses sont sous-estimées dans le jet : l'interface air-eau située sous le jet à la base du tube crée une ombre qui, d'une part ne permet pas un éclairage optimum du jet, et qui, d'autre part se déplace avec l'inclinaison du profil du tube, induisant ainsi des erreurs de vitesse. 

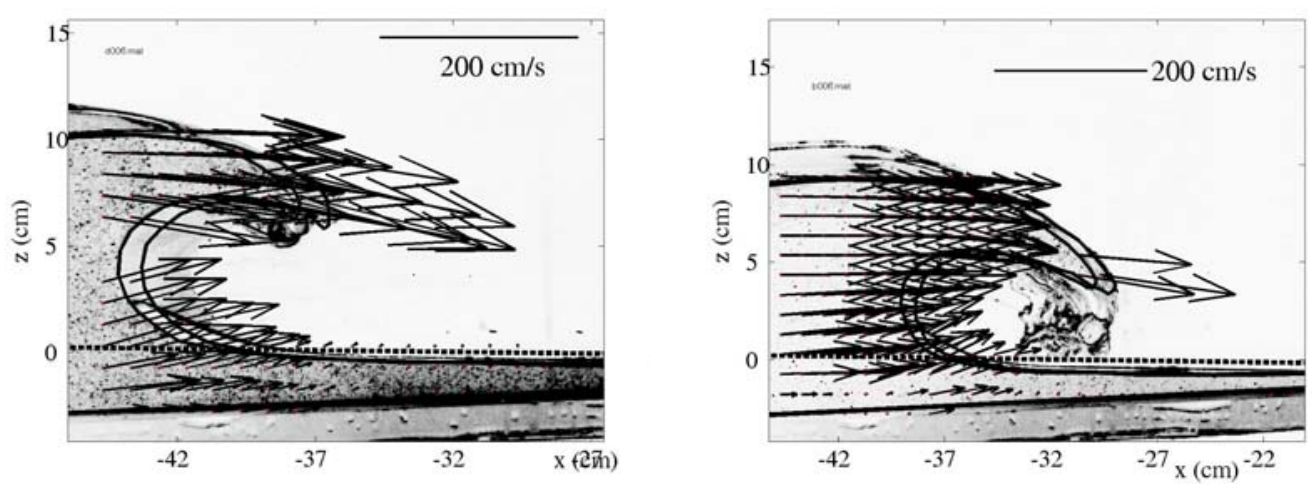

Figure 2 : exemples de champs de vitesse par mesure PIV (un vecteur sur quatre est représenté).

Les résultats expérimentaux (profils temporels, spatiaux et champs de vitesse) serviront de base de comparaison pour les modèles exposés ci-après.

\section{Présentation des méthodes numériques}

\subsection{Méthodologies basées sur les équations de Navier-Stokes}

Le problème considéré est l'écoulement de deux fluides incompressibles non miscibles. Pour un maillage structuré fixe non conforme à l'interface, la formulation eulérienne régissant l'écoulement diphasique dans les deux phases est composé des équations de Navier-Stokes (Eqs. 2), couplées avec la condition d'incompressibilité (Eq. 1).

$$
\left\{\begin{array}{c}
\rho\left(\frac{\partial \mathbf{U}}{\partial t}+(\mathbf{U} \cdot \nabla) \cdot \mathbf{U}\right)= \\
\rho \mathbf{g}-\nabla p+\nabla \cdot \mu\left(\nabla \mathbf{U}+\nabla^{T} \mathbf{U}\right)+\sigma \kappa \delta_{i} \mathbf{n}_{i} \\
\frac{\partial C}{\partial t}+\mathbf{U} \cdot \nabla C=0
\end{array}\right.
$$

$\rho$ et $\mu$ étant respectivement la densité et la viscosité dynamique, $\mathbf{U}$ le champ de vitesse, $\mathrm{p}$ le champ de pression, $\mathbf{g}$ le vecteur gravité, $\sigma$ la tension de surface, constante sur l'interface liquide - gaz, et $\kappa$ le rayon de courbure de l'interface. Les traitements particuliers des conditions aux limites sont développés en fonction des codes numériques présentés ci-après par chacune des équipes.

Les densités et viscosités, considérées comme constantes dans chacune des deux phases, sont des fonctions du point, que l'on détermine à partir de la position de l'interface entre les deux fluides. Celle-ci est décrite par une fonction binaire $\mathrm{C}$ traduisant le coefficient de remplissage de l'eau dans chaque volume de discrétisation. On définit ainsi $C$, telle que $C=1$ si le point est dans l'eau, et $\mathrm{C}=0$ si le point est dans l'air. L'interface est alors considérée comme étant représentée par l'iso-valeur $\mathrm{C}=0,5$. La valeur moyenne de la fonction $\mathrm{C}$ sur un volume de contrôle représente donc la fraction volumique d'eau présente dans ce volume. L'interface étant une surface matérielle et $\mathrm{C}$ une fonction caractéristique de cette interface, l'équation d'évolution temporelle de $\mathrm{C}$ est décrite par l'équation 3.

Les simulations présentées par les différentes équipes ne font pas intervenir de modélisation de la turbulence. Les images sont issues de la simulation directe des équations de Navier-Stokes (Eq. 2). 
L'hypothèse est faite que, durant toute la propagation de l'onde, jusqu'à son retournement, l'écoulement conserve un caractère irrotationnel. Les comparaisons qui seront effectuées concerneront des grandeurs macroscopiques, telles que l'abscisse du déferlement, la taille du jet et l'abscisse de son impact. A la conclusion des premières confrontations entre résultats numériques et expérimentaux, l'opportunité d'implémenter des modèles de turbulence sera étudiée.

\subsubsection{Laboratoire IMFT}

Une méthode VOF sans reconstruction d'interface est développée à l'IMFT (Benkenida et Magnaudet, 1999), pour l'étude d'écoulements diphasiques. Comme les méthodes VOF usuelles, l'écoulement à deux phases incompressibles est gouverné par le système d'équations 1 et 2 . Le dernier terme de l'équation de conservation de la quantité de mouvement (Eq. 2) est résolu en utilisant une formulation continue (Brackbill, Kothe et Zemach, 1992). La forme et la topologie de l'interface sont gouvernées par l'évolution de la fonction $\mathrm{C}$ satisfaisant l'équation 3. On peut interpréter $\mathrm{C}$ comme étant comme la fraction de volume locale de l'un des deux fluides (1 pour la phase liquide). La densité et la viscosité dynamique sont calculées dans les deux phases par:

$$
\begin{aligned}
& \rho=\rho_{1} C+(1-C) \rho_{2} \\
& \mu=\mu_{1} C+(1-C) \mu_{2}
\end{aligned}
$$

Les équations (2) et (3) sont résolues par une méthode de volumes finis du second ordre en espace. L'advection et les termes visqueux de (2) sont résolus par un algorithme semiimplicite ayant une précision du second ordre en temps, tandis que l'incompressibilité (Eq. 1) est satisfaite à la fin de chaque pas de temps, par une inversion directe de l'équation de Poisson 2D pour un potentiel auxiliaire. L'équation 3 est résolue en utilisant un schéma de Zalesak (1979) pour des équations hyperboliques. Ce schéma permet une correction du transport des flux. L'équation 3 est projetée suivant les deux directions, pour lesquelles le problème monodimensionnel est résolu de manière découplée.

Des simulations ont été réalisées pour une onde solitaire se propageant dans un domaine rectangulaire ayant tout d'abord un fond plat, puis à partir de $\mathrm{x}=0 \mathrm{~m}$, une plage à pente constante de $1: 10$. Le niveau de l'eau au repos est $\mathrm{d}=0,5 \mathrm{~m}$. La condition initiale est une onde solitaire approximée au neuvième ordre (Fenton, 1972) d'amplitude $\mathrm{a}=0,25 \mathrm{~m}$, initialement centrée en $\mathrm{x}=-2,5 \mathrm{~m}$. Le calcul est réalisé sur un maillage curviligne qui épouse la forme du fond. Les simulations montrent que la vague se propage sans se déformer jusqu'à la plage, où sa face avant se déforme. On peut voir sur la figure 3 la face avant de la vague se raidir. La vague s'effondre ensuite en produisant une région de mélange intense.

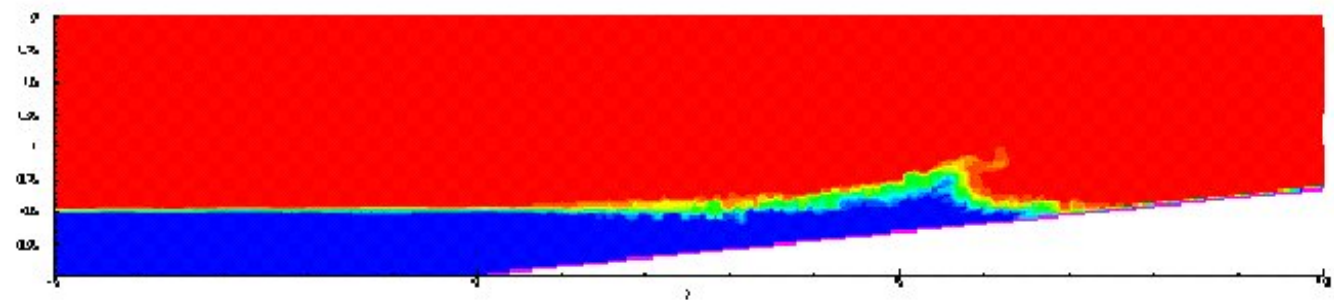

Figure 3: déferlement d'un soliton sur une plage à pente constante $-0<C<1$. 


\subsubsection{Laboratoire IRPHE}

L'IRPHE dispose d'un code numérique VOF aux différences finies, développé par S. Guignard à partir du noyau central du code SURFER (Lafaurie et al., 1994). La résolution des équations de Navier-Stokes discrétisées se fait par une méthode de projection. Le maillage est régulier, horizontal, décalé avec des nœuds alternés de pression et de vitesse. Le schéma temporel est explicite et d'ordre 1. Le schéma spatial est d'ordre 2 à l'intérieur du domaine sauf à l'interface. Le suivi d'interface est réalisé par la méthode de Construction de l'Interface Affine par Morceaux (CIAM-PLIC) (Li, 1995). Les conditions appliquées aux bords du domaine sont des conditions de non pénétration et de glissement sur les frontières solides, et avec sortie libre sur le toit du domaine.

Nous avons appliqué ce modèle à la propagation d'un soliton de type Tanaka (1986) sur des marches rectangulaires immergées de différentes hauteurs. La géométrie typique du bassin numérique est de $50 \mathrm{~m}$ de long, avec une profondeur d'eau de $1 \mathrm{~m}$, une hauteur initiale de soliton de $0,3 \mathrm{~m}$. Le pas d'espace de grille est de $0,050 \mathrm{~m}$ en horizontal et de 0,025 m en vertical. Le soliton est d'abord propagé sur quelques itérations par le modèle intégral potentiel BEM de Grilli et al. (1992), pour obtenir ainsi les conditions initiales du modèle VOF. Les codes Tanaka et BEM ont été fournis par S. Grilli. Quand la hauteur de la marche sous-marine dépasse un certain seuil, le soliton déferle (déferlement de type ressaut hydraulique), le résultat principal étant la génération dans l'eau d'ondes de vorticité alternativement positive et négative, dans le sillage du soliton, comme l'illustre la figure 4. Le soliton génère également un vortex dans l'air au dessus de son interface.

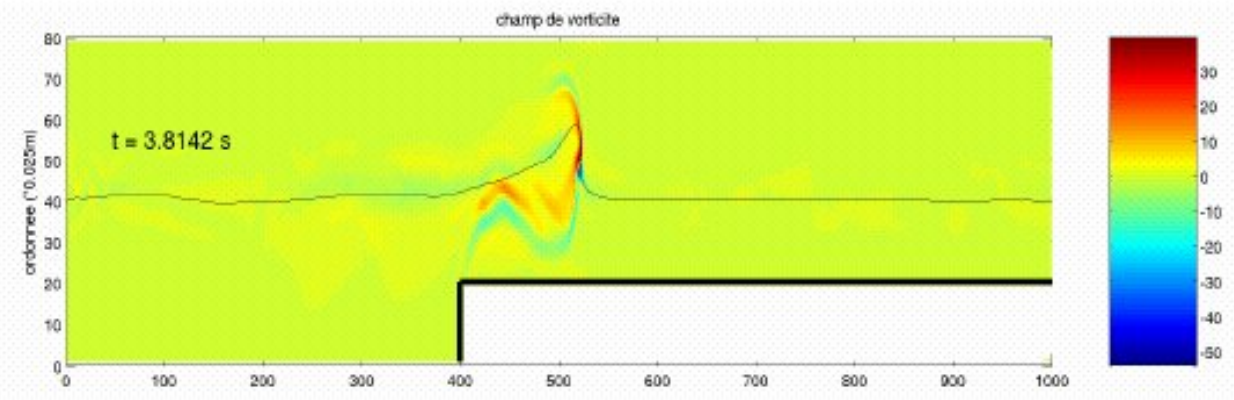

Figure 4 : propagation d'un soliton sur une marche immergée - champ de vorticité (en s ${ }^{-1}$ ).

\subsubsection{Laboratoire LSEET}

La méthode de suivi de surface libre utilisée, méthode SL -VOF, est couplée avec le logiciel de CFD EOLE développé par PRINCIPIA. Le code EOLE utilise une approche de pseudo-compressibilité (Viviand, 1980, De Jouëtte et al., 1991) pour résoudre les équations de Navier-Stokes. La méthode VOF est une méthode de type semi-lagrangienne, SL -VOF (Segment Lagrangian Volume of Fluid Method) en 2D (Guignard, 2001) et en 3D (Biausser, 2003). L'interface est modélisée avec le concept PLIC (Piecewise Linear Interface Calculation), c'est-à-dire qu'elle est représentée par une fonction affine par morceaux. La nouvelle position de la surface libre est réactualisée à partir des champs de vitesse calculés par le solveur Navier-Stokes d'EOLE lors d'une itération temporelle. L'advection des plans est effectuée par un schéma lagrangien d'ordre 1 et le nouveau champ de VOF est déduit de la position des segments. Ceci permet l'utilisation de pas de temps plus élevés et donc de diminuer les temps de calcul, ce qui s'avère crucial pour des calculs 3D. On utilise une formulation curviligne des équations de bilan, ce qui permet de traiter de grandes déformations de l'interface. 
Ainsi, le cas du déferlement d'un soliton sur un fond de type bosse a été étudié. La géométrie du fond est la même que celle utilisée par Grilli et al. (2001). Afin de limiter les temps de calcul, le couplage avec le code BEM est utilisé comme initialisation du modèle Navier-Stokes / VOF jusqu'à la phase de retournement.
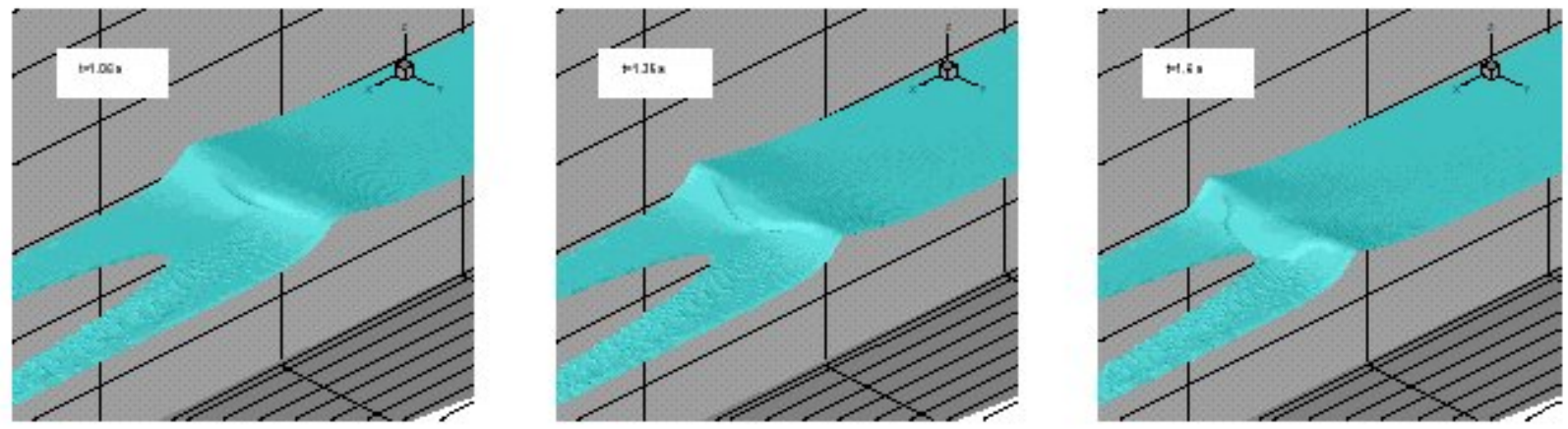

Figure 5: évolution du déferlement $-C=0,5$.

\subsubsection{Laboratoire TREFLE}

Le logiciel utilisé, Aquilon, est développé au laboratoire TREFLE. La méthode du Lagrangien Augmenté variable (Vincent et al., 2004) est mise en oeuvre pour résoudre le couplage vitesse / pression dans le système d'équations présenté auparavant (Eqs. 1 à 2). La discrétisation en temps est réalisée par un schéma d'Euler d'ordre deux (schéma de Gear), tandis qu'un schéma hybride Centré - Décentré Amont est utilisé pour les termes convectifs non-linéaires. Un schéma centré du second ordre est choisi pour l'approximation des termes visqueux. Avec la même approche que l'IMFT, le suivi d'interface est réalisé par une méthode VOF sans reconstruction d'interface, appelée TVD Lax-Wendroff (Vincent et Caltagirone, 2000). Celle-ci est d'une grande souplesse d'utilisation et permet d'étudier librement les problèmes de déconnections et reconnections d'interfaces tels qu'on les rencontre dans le déferlement des ondes côtières.

Des travaux ont déjà fait l'objet de publications (Lubin et al., 2003), telle que l'étude tridimensionnelle de la génération de structures turbulentes par le déferlement d'une onde sinusoïdale instable de grande amplitude dans un domaine périodique. De plus, dans le cadre du programme national PATOM, l'étude du déferlement d'ondes solitaires sur plages inclinées ou sur récifs immergés a été menée (déferlements plongeants ou déversants). La propagation d'onde solitaire sur fonds plats ou sur des obstacles immergés a permis de vérifier la bonne précision de nos méthodes et leur aptitude à décrire précisément les phénomènes. La figure suivante 6 présente l'exemple du déferlement d'une onde solitaire d'amplitude $\mathrm{H}=0,5 \mathrm{~m}$ initialisée sur un fond plat situé à $1 \mathrm{~m}$ de profondeur. Celle-ci déferle sur une plage inclinée de pente $1: 15$.

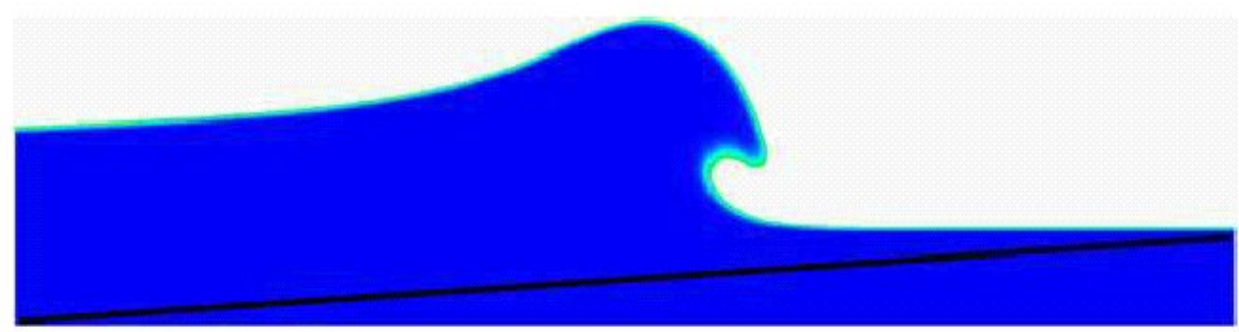

Figure 6: déferlement plongeant d'une onde solitaire sur plage inclinée - $C>0,5$. 


\subsection{Méthodologie basée sur les éléments frontières}

Les méthodes numériques basées sur les éléments frontières (BEM - Boundary Element Method) ont prouvé leur efficacité et leur précision quant à la résolution des équations régissant l'évolution des écoulements potentiels non linéaires à surface libre. Les modèles bi(2D) et tri- (3D) dimensionnels de génération et de propagation de houle (Canaux à Houle Numériques - CAN) présentent l'avantage de fournir une réponse rapide à des problèmes d'ingénierie ou de recherche dans le domaine côtier, et se montrent très peu coûteuses en temps de calcul comparativement à d'autres méthodes où le domaine est complètement discrétisé.

Le modèle 2D BEM développé par Grilli et al. $(1989,2001)$ est utilisé pour ces simulations. Le canal expérimental est reproduit numériquement, ainsi que le batteur de houle générant les ondes solitaires utilisées dans ces expériences. Cependant, la particularité de l'installation expérimentale a nécessité des développements pour prendre en compte le fait que le pivot du batteur se situait sous le fond du canal, y compris la frontière courbe à la base du mécanisme (Fig. 1). La course du batteur expérimental est reproduite dans le canal à houle numérique grâce au même algorithme que celui utilisé lors de la campagne de mesures pour faire fonctionner le piston hydraulique. Ainsi, les dimensions géométriques du canal sont spécifiées dans les simulations numériques, reproduisant en particulier les variations du fond grâce aux deux plages de pente $1: 6$ et $1: 15$.

Du fait de différences observées entre les profils initiaux générés dans le CAN et les expériences, ces simulations ont dû faire l'objet de tests de sensibilité et de modification de la loi de batteur. En effet, une étude approfondie de la génération de la houle a montré que de légers changements dans la course du batteur pouvaient produire des différences non négligeables quant aux profils des ondes générées. Une fois ces approximations prises en compte, les figures suivantes (Figs. 7 et 8) montrent le bon accord observé entre les résultats numériques et les profils expérimentaux mesurés dans la zone de déferlement.
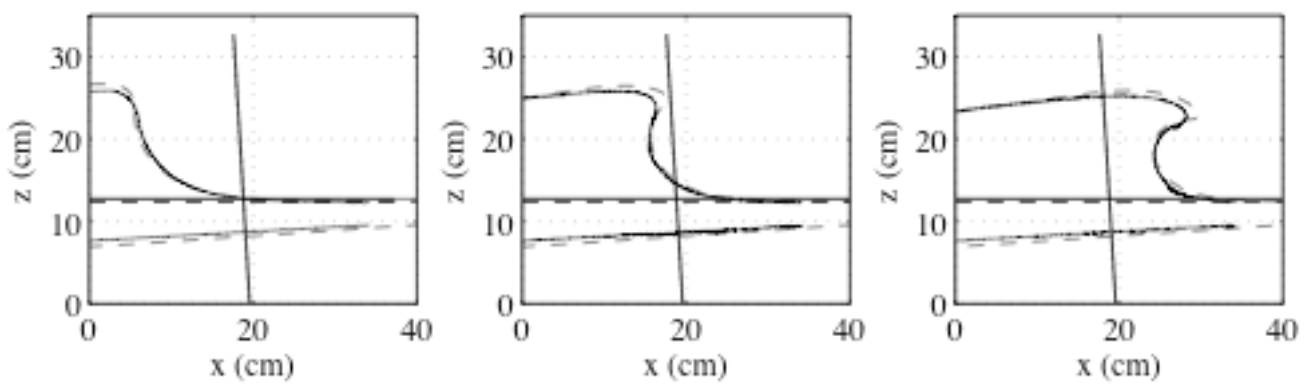

Figure 7: Comparaison entre profils issus de la simulation numérique BEM ( --- ) et visualisations expérimentales (-) amplitude initiale $H=0,1 \mathrm{~m}$, profondeur $\mathrm{h}=0,74 \mathrm{~m}$.
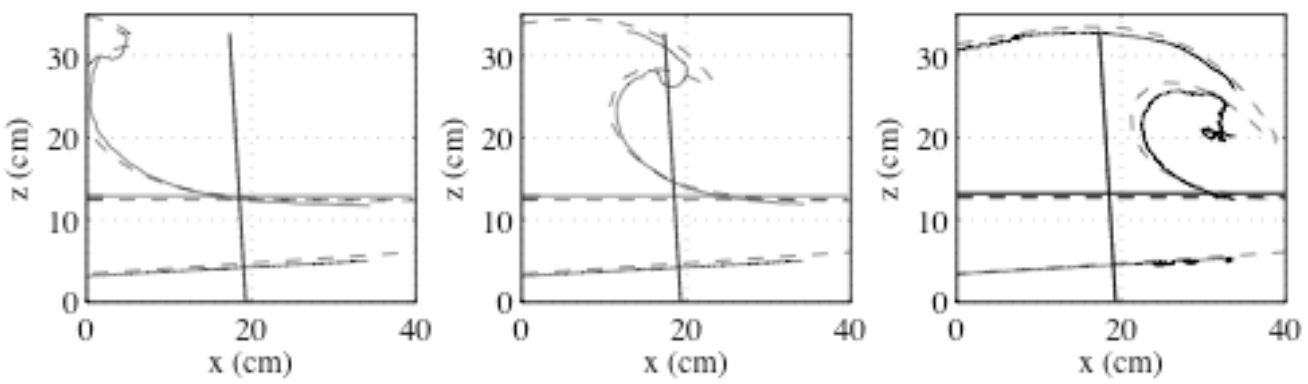

Figure 8: Comparaison entre profils issus de la simulation numérique BEM ( --- ) et visualisations expérimentales ( - amplitude initiale $H=0,16 \mathrm{~m}$, profondeur $h=0,76 \mathrm{~m}$. 


\section{Conclusion et perspectives}

Nous avons présenté un exercice de validation et de comparaisons en cours au sein du programme national PATOM. Ce travail réunit différents laboratoires amenant chacun ses outils de simulation dans le but de s'inscrire dans un effort de recherche orienté vers l'amélioration des méthodes numériques servant à la description du phénomène du déferlement.

Le couplage des champs de données issus des simulations réalisées avec le code BEM et les codes Navier-Stokes sera effectué à différents instants. La propagation sur fond plat, la déformation à l'approche de la zone de déferlement, le retournement de l'onde, comprenant la génération et l'impact du jet déferlant, seront les principales étapes faisant l'objet de comparaisons. Cette phase de l'exercice est en cours et les résultats et leurs discussions seront présentés lors de la conférence.

\section{Remerciements}

Les auteurs tiennent à remercier le Programme National sur l'Atmosphère et l'Océan Multi-échelles (PATOM) pour son soutien financier, et plus particulièrement Hervé Michallet, chargé de recherche au Laboratoire des Ecoulements Géophysiques et Industriels (LEGI) de Grenoble, et responsable scientifique du thème «Hydrodynamique des vagues en zone de déferlement » pour son implication et sa disponibilité.

\section{Références}

1.Adrian, R., (1991), Particle imaging techniques for experimental fluid mechanics, Annual Review Fluid Mech., 23, 261-304.

2.Benkenida, A. and Magnaudet, J. (1999). Une méthode de simulation d'écoulement diphasiques sans reconstruction d'interfaces, C. R. Acad. Sci. Paris, Ser. IIb, 328, 25-32.

3.Biausser, B. (2003). Suivi d'interface tridimensionnel de type Volume of Fluid: application au déferlement, Thèse de doctorat, Université de Toulon et du Var.

4.Brackbill, J. U., Kothe, D. B. and Zemach, C. (1992). A continuum method for modeling surface tension, J. Comput. Phys., 100, 335-354.

5.De Jouëtte, C., Viviand, H., Wormon, S., Le Gouez, J.M. (1991). Pseudocompressibility Methods for Incompressible Flow Calculation, Proc $4^{\text {th }}$ Intenational Symposium on Computational Fluid Dynamics, University of California, Davis. 71.

6.Fenton, J. (1972). A ninth-order solution for the solitary wave, J. Fluid Mech., 53, 257-

7.Grilli, S. T., Skourup, J., and Svendsen, I. A. (1989). An efficient boundary element method for nonlinear waves, Engng. Analysis with Boundary Elements, 6 (2), 97-107.

8.Grilli, S. T., Losada, M., and Martin, F. (1992). The breaking of a solitary wave over a step: modeling and experiments", E. Hydraulic engineering software IV., Fluid flow modelling, 1, 575-586.

9.Grilli, S. T., Guyenne, P., and Dias, F. (2001), A fully nonlinear model for threedimensional overturning waves over arbitrary bottom, Intl J.Numeric. Meth. Fluids, 35 (1), 829-867.

10.Grilli, S. T., Gilbert, R., Lubin, P., Vincent, S., Astruc, D., Legendre, D., Duval, M., Kimmoun, O., Branger, H., Devrard, D., Fraunié, P., Abadie, S. (2004). Numerical modeling and experiments for solitary wave shoaling and breaking over sloping beach, Proc. $14^{\text {th }}$ International Offshore and Polar Engineering Conference and Exhibition, ISOPE 2004, Toulon, France. 
11.Guignard, S. (2001). Suivi d'interface de type VOF : application au déferlement des ondes de gravité dû aux variations bathymétriques, thèse de doctorat de l'Université de Toulon et du Var, UFR Sciences et Techniques.

12.Kimmoun, O., Branger, H., and Zucchini, B. (2004). Laboratory PIV measurements of wave breaking on a beach., Proc. $14^{\text {th }}$ International Offshore and Polar Engineering Conference and Exhibition, ISOPE 2004, Toulon, France.

13.Lafaurie, B., Nardone, C., Scardovelli, R., Zaleski, S., and Zanetti, G. (1994). Modelling merging and fragmentation in multiphase flows with SURFER, J. Comp. Phys., 113, 134-147.

14.Li J. (1995). Calcul d'interface affine par morceaux (Piecewise Linear Interface Calculation), C. R. Acad. Sci. Paris, Ser. IIb, 320, 391 - 396.

15.Lubin, P., Vincent, S., Caltagirone, J.-P. and Abadie, S. (2003). Fully threedimensional direct numerical simulation of plunging breaking waves, C. R. Mécanique, 331, 495-501.

16.Meunier, P. and Leweke, T. (2003). Analysis and treatment of error due to high velocity gradients in particle image velocymetry, Exp. in Fluids, 35 (5), 408-421.

17.Vincent, S. and Caltagirone, J.-P. (2000). A one cell local multigrid method for solving unsteady incompressible multi-phase flows, J. Comput. Phys., 163, 172-215.

18.Vincent, S. and Caltagirone, J.-P. (2000). A one cell local multigrid method for solving unsteady incompressible multi-phase flows, J. Comput. Phys., 163, 172-215.

19.Vincent, S., Caltagirone, J.-P., Lubin, P. and Randrianarivelo, T. N. (2004). An adaptative augmented Lagrangian method for three-dimensional multi-material flows, Computers and Fluids (à paraitre).

20.Viviand, H. (1980). Pseudo-unsteady Methods for Transonic Flow Computations, $19^{\text {th }}$ International Conference on Numerical Methods in Fluid Dynamics, Stanford, in Lecture Notes in Physics, 141, Springer-Verlag, New-York.

21.Tanaka, M. (1986). The stability of solitary waves, Phys. Fluids, 29 (3), 650-655.

22.Zalesak, S. T. (1979). Fully multidimensional flux-corrected transport algorithm for fluids, J. Comput. Phys., 31, 335-359. 